Article

\title{
Positive Solutions of a Fractional Thermostat Model with a Parameter
}

\section{Xinan Hao * (D) and Luyao Zhang}

School of Mathematical Sciences, Qufu Normal University, Qufu 273165, China; shuxuequfu@126.com

* Correspondence: haoxinan2004@163.com

Received: 19 December 2018; Accepted: 17 January 2019; Published: 21 January 2019

check for updates

\begin{abstract}
We study the existence, multiplicity, and uniqueness results of positive solutions for a fractional thermostat model. Our approach depends on the fixed point index theory, iterative method, and nonsymmetry property of the Green function. The properties of positive solutions depending on a parameter are also discussed.
\end{abstract}

Keywords: positive solution; fractional thermostat model; fixed point index; dependence on a parameter

\section{Introduction}

In this paper, we investigate a fractional nonlocal boundary value problem (BVP)

$$
\left\{\begin{array}{l}
{ }^{c} D_{0+}^{\alpha} x(t)+\lambda g(t) f(x(t))=0, \quad t \in(0,1), \\
x^{\prime}(0)=0, \beta^{c} D_{0+}^{\alpha-1} x(1)+x(\eta)=0
\end{array}\right.
$$

where $1<\alpha \leq 2, \beta>0,0 \leq \eta \leq 1, \beta \Gamma(\alpha)>(1-\eta)^{\alpha-1},{ }^{c} D_{0+}^{\alpha}$ is the Gerasimov-Caputo fractional derivative of order $\alpha, \lambda>0$ is a parameter, $f \in C([0,+\infty),[0,+\infty)), g \in C((0,1),[0,+\infty))$, and $0<\int_{0}^{1} g(t) d t<+\infty$.

One motivation is that the thermostat model

$$
\left\{\begin{array}{l}
x^{\prime \prime}(t)+g(t) f(t, x(t))=0, \quad t \in(0,1) \\
x^{\prime}(0)=0, \beta x^{\prime}(1)+x(\eta)=0
\end{array}\right.
$$

which is a special case with $\alpha=2$ and $\lambda=1$, has been discussed by Infante and Webb [1,2]. They established multiplicity results of BVP (2). These types of problems have been investigated by various scholars, see References [3-17].

Recently, the thermostat model was extended to the fractional case

$$
\left\{\begin{array}{l}
{ }^{c} D_{0+}^{\alpha} x(t)+f(t, x(t))=0, \quad t \in(0,1), \alpha \in(1,2], \\
x^{\prime}(0)=0, \beta^{c} D_{0+}^{\alpha-1} x(1)+x(\eta)=0
\end{array}\right.
$$

where $\beta>0,0 \leq \eta \leq 1, f \in C([0,1] \times[0,+\infty),[0,+\infty))$. Nieto and Pimentel [18] proved the existence of positive solutions based on the Krasnosel'skii fixed point theorem. Cabada and Infante [19] discussed the multiplicity results of positive solutions for BVP (3).

In Reference [20], Shen, Zhou, and Yang studied a fractional thermostat model

$$
\left\{\begin{array}{l}
{ }^{c} D_{0+}^{\alpha} x(t)+\lambda f(t, x(t))=0, \quad t \in(0,1), 1<\alpha \leq 2, \\
x^{\prime}(0)=0, \beta^{c} D_{0+}^{\alpha-1} x(1)+x(\eta)=0,
\end{array}\right.
$$


where $\beta>0,0 \leq \eta \leq 1, \beta \Gamma(\alpha)>(1-\eta)^{\alpha-1}, \lambda>0, f:[0,1] \times[0,+\infty) \rightarrow[0,+\infty)$ is continuous. The authors obtained intervals of parameter $\lambda$ that correspond to at least one and no positive solutions. Similar fractional thermostat problems have been studied in References [21-24].

In this paper, we deal with positive solutions for the fractional thermostat model (1). The existence, multiplicity, and uniqueness results are established by the fixed point index theory and iterative method. The properties of positive solutions depending on a parameter are also discussed. Some of the ideas in this paper are from References [25,26]. Let us remark that the definition of the Gerasimov-Caputo derivative was first introduced and applied by Gerasimov in 1947 and then by Caputo in 1967, see for example, the overview by Novozhenova in Reference [27]. For details on the theory and applications of the fractional derivatives and integrals and fractional differential equations, see References [28-31].

\section{Preliminaries}

Lemma 1 ([20]). Given $u(t) \in C(0,1) \cap L^{1}(0,1)$, the solution of the problem

$$
\left\{\begin{array}{c}
{ }^{c} D_{0+}^{\alpha} x(t)+u(t)=0, \quad t \in(0,1), \\
x^{\prime}(0)=0, \beta^{c} D_{0+}^{\alpha-1} x(1)+x(\eta)=0
\end{array}\right.
$$

is

$$
x(t)=\int_{0}^{1} G(t, s) u(s) d s, \quad t \in[0,1]
$$

where

$$
G(t, s)=\left\{\begin{array}{l}
\beta-\frac{(t-s)^{\alpha-1}}{\Gamma(\alpha)}+\frac{(\eta-s)^{\alpha-1}}{\Gamma(\alpha)}, \quad 0 \leq s \leq \eta, s \leq t, \\
\beta+\frac{(\eta-s)^{\alpha-1}}{\Gamma(\alpha)}, \quad 0 \leq s \leq \eta, s \geq t \\
\beta-\frac{(t-s)^{\alpha-1}}{\Gamma(\alpha)}, \quad \eta \leq s \leq 1, s \leq t, \\
\beta, \quad \eta \leq s \leq 1, s \geq t
\end{array}\right.
$$

and $G(t, s)$ satisfies:

(i) $G(t, s):[0,1] \times[0,1] \rightarrow(0,+\infty)$ is continuous;

(ii) $\frac{\partial}{\partial t} G(t, s) \leq 0, t, s \in[0,1]$;

(iii) $\gamma \bar{G}=\underline{G} \leq G(1, s) \leq G(t, s) \leq G(0, s) \leq \bar{G}, \quad t, s \in[0,1]$,

where

$$
\gamma=\frac{\beta \Gamma(\alpha)-(1-\eta)^{\alpha-1}}{\beta \Gamma(\alpha)+\eta^{\alpha-1}}, \quad \underline{G}=\frac{\beta \Gamma(\alpha)-(1-\eta)^{\alpha-1}}{\Gamma(\alpha)}, \quad \bar{G}=\frac{\beta \Gamma(\alpha)+\eta^{\alpha-1}}{\Gamma(\alpha)} .
$$

Denote $E=C[0,1]$ and $\|x\|=\sup _{t \in[0,1]}|x(t)|$. We define the cone

$$
P=\left\{x \in E: x(t) \geq 0, \inf _{t \in[0,1]} x(t) \geq \gamma\|x\|\right\} .
$$

For any $0<r<+\infty$, let $P_{r}=\{x \in P:\|x\|<r\}$. We define $T:(0,+\infty) \times E \rightarrow E$ as

$$
T(\lambda, x)(t)=\lambda \int_{0}^{1} G(t, s) g(s) f(x(s)) d s, \quad t \in[0,1] .
$$

It is obvious from Lemma 1 that if $x \in P$ is a fixed point of operator $T$, then $x$ is a positive solution of Problem (1). By regularity arguments, we can show that $T$ is completely continuous and $T(P) \subset P$. 
Define the linear operator $L: E \rightarrow E$ by

$$
L x(t)=\int_{0}^{1} G(t, s) g(s) x(s) d s, \quad t \in[0,1] .
$$

By the Krein-Rutman theorem, we see that the spectral radius $r(L)$ of the operator $L$ is positive, and $L$ has positive eigenfunction $\varphi_{1}$ corresponding to its first eigenvalue $\mu_{1}=(r(L))^{-1}$.

Lemma 2 ([32]). Let $P$ be a cone in Banach space E. Suppose that $T: P \rightarrow P$ is a completely continuous operator. (i) If $T u \neq \mu u$ for any $u \in \partial P_{r}$ and $\mu \geq 1$, then $i\left(T, P_{r}, P\right)=1$. (ii) If $T u \neq u$ and $\|T u\| \geq\|u\|$ for any $u \in \partial P_{r}$, then $i\left(T, P_{r}, P\right)=0$.

Denote

$$
f_{0}=\lim _{s \rightarrow 0} \frac{f(s)}{s}, \quad f_{\infty}=\lim _{s \rightarrow \infty} \frac{f(s)}{s}, \quad A=\int_{0}^{1} G(0, s) g(s) d s, \quad l=\min _{s \in(0, \infty)} \frac{f(s)}{s} .
$$

We assume that:

$\left(H_{1}\right) f$ is nondecreasing on $[0,+\infty)$;

$\left(H_{2}\right)$ there exists a function $\phi:(0,1] \rightarrow[0,1]$ continuous nondecreasing, such that $f(\kappa x) \geq \phi(\kappa) f(x)$ for $0<\kappa<1, x>0$, and $F(\kappa):=\frac{\kappa}{\phi(\kappa)}$ is strictly increasing on $(0,1]$ and $F(1)=1$.

Lemma 3. Suppose that $\left(H_{1}\right)$ holds, $f_{0}=\infty$ and $l>0$. If $0<\lambda_{1}<\lambda_{2}<\frac{1}{l A}$, then there exist $x_{1}, x_{2} \in$ $P \backslash\{\theta\}, x_{1} \leq x_{2}$, such that $T\left(\lambda_{1}, x_{1}\right)(t)=x_{1}(t)$ and $T\left(\lambda_{2}, x_{2}\right)(t)=x_{2}(t)$.

Proof. Assume $s_{0} \in(0, \infty)$ such that $f\left(s_{0}\right)=l_{s_{0}}$. Since $0<\lambda_{1}<\lambda_{2}<\frac{1}{l A}$, we have $l<\frac{1}{\lambda_{2} A}<\frac{1}{\lambda_{1} A}$. We define

$$
x_{0}(t)=\frac{s_{0}}{A} \int_{0}^{1} G(t, s) g(s) d s, \quad t \in[0,1],
$$

then

$$
\left\|x_{0}\right\|=x_{0}(0)=s_{0}, \quad x_{0}(t) \geq \frac{s_{0}}{A} \int_{0}^{1} \gamma G(0, s) g(s) d s=\gamma\left\|x_{0}\right\|, \quad t \in[0,1] .
$$

Therefore, $x_{0} \in P$ and $\left\|x_{0}\right\|=s_{0}$. Direct computations yield

$$
\begin{aligned}
T\left(\lambda_{1}, x_{0}\right)(t) & =\lambda_{1} \int_{0}^{1} G(t, s) g(s) f\left(x_{0}(s)\right) d s \leq \lambda_{1} \int_{0}^{1} G(t, s) g(s) f\left(\left\|x_{0}\right\|\right) d s \\
& =\lambda_{1} l_{0} \int_{0}^{1} G(t, s) g(s) d s<\frac{s_{0}}{A} \int_{0}^{1} G(t, s) g(s) d s=x_{0}(t), \quad t \in[0,1] .
\end{aligned}
$$

Define

$$
x_{1}^{1}(t)=T\left(\lambda_{1}, x_{0}\right)(t), x_{1}^{j}(t)=T\left(\lambda_{1}, x_{1}^{j-1}\right)(t)=T^{j}\left(\lambda_{1}, x_{0}\right)(t), \quad j=2,3, \cdots, t \in[0,1] .
$$

Direct calculations show that $x_{0}>x_{1}^{1}>x_{1}^{2}>\cdots>x_{1}^{j}>x_{1}^{j+1}>\cdots \geq \theta$. Hence, sequence $\left\{x_{1}^{j}\right\}_{j=1}^{\infty}$ is decreasing and bounded from below, $\lim _{j \rightarrow \infty} x_{1}^{j}(t)$ exists and convergence is uniform for $t \in[0,1]$. Assume that $\lim _{j \rightarrow \infty} x_{1}^{j}=x_{1}$, we claim that $x_{1}(t)>0$. Otherwise, since $x_{1} \in P, x_{1}(t)=0$, i.e., $\lim _{j \rightarrow \infty} x_{1}^{j}(t)=0, t \in[0,1]$, and hence from $x_{1}^{j} \in P$, we deduce $\left\|x_{1}^{j}\right\| \rightarrow 0$. Since $f_{0}=\infty$, for any $H>\frac{1}{\lambda_{1} \gamma \mathrm{A}}$, there is integral $Z>0$ such that for $j>Z$, we have $f\left(x_{1}^{j}(t)\right)>H x_{1}^{j}(t)$, and hence 


$$
\begin{aligned}
x_{1}^{j+1}(0) & =\lambda_{1} \int_{0}^{1} G(0, s) g(s) f\left(x_{1}^{j}(s)\right) d s \\
& >\lambda_{1} H \gamma \int_{0}^{1} G(0, s) g(s)\left\|x_{1}^{j}\right\| d s \\
& \geq x_{1}^{j}(0) \lambda_{1} H \gamma A>x_{1}^{j}(0) .
\end{aligned}
$$

The contradiction shows that $x_{1} \in P \backslash\{\theta\}$ and $x_{1}=T\left(\lambda_{1}, x_{1}\right)$.

Similarly, from $x_{2}^{1}(t)=T\left(\lambda_{2}, x_{0}\right)(t)$ and $x_{2}^{j}(t)=T\left(\lambda_{2}, x_{2}^{j-1}\right)(t), j=2,3, \cdots$, we deduce

$$
x_{0}>x_{2}^{1}>x_{2}^{2}>\cdots>x_{2}^{j}>x_{2}^{j+1}>\cdots \geq \theta,
$$

$\lim _{j \rightarrow \infty} x_{2}^{j}=x_{2} \in P \backslash\{\theta\}$, and $x_{2}=T\left(\lambda_{2}, x_{2}\right)$. It follows from $x_{1}^{1}=T\left(\lambda_{1}, x_{0}\right)<T\left(\lambda_{2}, x_{0}\right)=x_{2}^{1}$ and the monotonicity of $f$ that $x_{1}^{j} \leq x_{2}^{j}, j=2,3, \cdots$. Therefore, $x_{1} \leq x_{2}$.

Lemma 4. If $f_{\infty}=\infty$, then for any $\mu>0$, the set $S_{\mu}=\{x \in P: T(\lambda, x)=x, \lambda \in[\mu, \infty)\}$ is bounded.

Proof. Otherwise, there exists $x_{n} \in S_{\mu}$ corresponding to $\lambda_{n} \in[\mu, \infty)$ such that

$$
T\left(\lambda_{n}, x_{n}\right)=x_{n}, \quad \lim _{n \rightarrow \infty}\left\|x_{n}\right\|=\infty .
$$

Because $f_{\infty}=\infty$, there is $X>0$ such that $f(s)>H s$ for $s>X$, where $H>\frac{1}{\mu \gamma A}$. Since $\lim _{n \rightarrow \infty}\left\|x_{n}\right\|=\infty$, there exists $N_{0}>0$ such that $\left\|x_{n}\right\|>\frac{X}{\gamma}$ for $n>N_{0}$, and $x_{n}(t) \geq \gamma\left\|x_{n}\right\|>X, t \in$ $[0,1]$. Then, for any $n>N_{0}$, we obtain

$$
\left\|x_{n}\right\|>\lambda_{n} \int_{0}^{1} G(0, s) g(s) H x_{n}(s) d s>\mu H \gamma\left\|x_{n}\right\| A>\left\|x_{n}\right\|,
$$

which is absurd, and hence $S_{\mu}$ is bounded.

Lemma 5. Assume that $\left(H_{1}\right)$ holds, and that $f_{0}=f_{\infty}=\infty$. Then, $T$ admits a fixed point for $\lambda=\frac{1}{l A}$.

Proof. Choosing a sequence $0<\lambda_{1}<\lambda_{2}<\cdots<\lambda_{n}<\lambda_{n+1}<\cdots<\frac{1}{l A}$ such that $\lim _{n \rightarrow \infty} \lambda_{n}=\frac{1}{l A}$. By Lemma 3, there exists a nondecreasing sequence $\left\{x_{n}\right\}_{n=1}^{\infty} \subset P \backslash\{\theta\}$ such that $x_{n}=T\left(\lambda_{n}, x_{n}\right)$. By Lemma 4, we know that $\left\{x_{n}\right\}_{n=1}^{\infty}$ is uniformly bounded and equicontinuous. By using the Arzela-Ascoli theorem, we can prove that there exists $\left\{x_{n_{k}}\right\}_{k=1}^{\infty} \subset\left\{x_{n}\right\}_{n=1}^{\infty}$ such that $x_{n_{k}} \rightarrow \widetilde{x} \in E$ uniformly on $[0,1]$. Therefore, $x_{n_{k}}$ satisfies

$$
x_{n_{k}}(t)=T\left(\lambda_{n_{k}}, x_{n_{k}}\right)(t)=\lambda_{n_{k}} \int_{0}^{1} G(t, s) g(s) f\left(x_{n_{k}}(s)\right) d s, \quad t \in[0,1] .
$$

Passing to the limit as $k \rightarrow \infty$, we obtain

$$
\widetilde{x}(t)=\frac{1}{l A} \int_{0}^{1} G(t, s) g(s) f(\widetilde{x}(s)) d s, \quad t \in[0,1] .
$$

Hence, $\widetilde{x}=T\left(\frac{1}{l A}, \widetilde{x}\right)$.

Lemma 6. Assume that $\left(H_{1}\right)$ holds, and that $f(0)>0$. Then, for any $x \in P$, there exist $U_{x} \geq V>0$ such that

$$
V K_{\lambda} \leq T(\lambda, x)(t) \leq U_{x} K_{\lambda}, \quad t \in[0,1],
$$

where

$$
K_{\lambda}=\lambda \int_{0}^{1} g(t) d t
$$


Proof. By $\left(H_{1}\right)$, for any $x \in P$ and $t \in[0,1]$, we have

$$
T(\lambda, x)(t) \geq \underline{G} f(0) \lambda \int_{0}^{1} g(t) d t:=V K_{\lambda}
$$

and

$$
T(\lambda, x)(t) \leq \bar{G} f(\|x\|) \lambda \int_{0}^{1} g(t) d t:=U_{x} K_{\lambda}
$$

\section{Main Results}

Theorem 1. Assume that $f_{\infty}=\infty$ and $0<f_{0}<\infty$. Then, for any $0<\lambda<\frac{\mu_{1}}{f_{0}}, B V P$ (1) admits a positive solution.

Proof. Since $0<\lambda<\frac{\mu_{1}}{f_{0}}$, there exist $\varepsilon>0$ small enough and $r>0$ such that $\lambda\left(f_{0}+\varepsilon\right)<\mu_{1}$, and $\frac{f(s)}{s}<f_{0}+\varepsilon$ for $s \in(0, r]$. We claim that

$$
T(\lambda, x) \neq \mu x, \quad x \in \partial P_{r}, \mu \geq 1 .
$$

Otherwise, there exist $x_{0} \in \partial P_{r}$ and $\mu_{0} \geq 1$ such that $T\left(\lambda, x_{0}\right)=\mu_{0} x_{0}$. Since $0<\gamma r \leq x_{0}(t) \leq$ $\left\|x_{0}\right\|=r$, we have

$$
\mu_{0} x_{0}(t) \leq \lambda\left(f_{0}+\varepsilon\right) \int_{0}^{1} G(t, s) g(s) x_{0}(s) d s=\lambda\left(f_{0}+\varepsilon\right) L x_{0}(t),
$$

then $L x_{0}(t) \geq \frac{\mu_{0}}{\lambda\left(f_{0}+\varepsilon\right)} x_{0}(t)$. Thus, $r(L) \geq \frac{\mu_{0}}{\lambda\left(f_{0}+\varepsilon\right)} \geq \frac{1}{\lambda\left(f_{0}+\varepsilon\right)}$. It follows that $\mu_{1} \leq \lambda\left(f_{0}+\varepsilon\right)$, which is a contradiction. Then, $i\left(T, P_{r}, P\right)=1$.

Next, we prove that $i\left(T, P_{R}, P\right)=0$ for some $R>r$. In fact, $f_{\infty}=\infty$ implies that $f(s)>M s$ for some large $R_{1}>0$ and $s \geq R_{1}$, where $M>(\lambda \gamma A)^{-1}$. Let $R>\max \left\{r, \frac{R_{1}}{\gamma}\right\}$. For $x \in \partial P_{R}$, we have $x(t) \geq \gamma\|x\|=\gamma R>R_{1}, t \in[0,1]$, then

$$
\|T(\lambda, x)\| \geq \lambda M \int_{0}^{1} G(0, s) g(s) x(s) d s \geq \lambda M \gamma\|x\| A>\|x\| .
$$

Hence, $i\left(T, P_{R}, P\right)=0$, and $i\left(T, P_{R} \backslash \bar{P}_{r}, P\right)=-1$. Therefore, $T$ admits a fixed point $x^{*} \in P_{R} \backslash \bar{P}_{r}$.

Theorem 2. Assume that $\left(H_{1}\right)$ holds, and that $f_{0}=f_{\infty}=\infty$. Then, BVP (1) has at least one and two positive solutions for $\lambda=\frac{1}{l A}$ and $\lambda \in\left(0, \frac{1}{l A}\right)$, respectively.

Proof. By Lemma 5, BVP (1) admits a positive solution for $\lambda=\frac{1}{l A}$. For $\lambda \in\left(0, \frac{1}{l A}\right)$, by Lemmas 3 and 5 , there exist $\tilde{x}, x_{\lambda} \in P \backslash\{\theta\}, x_{\lambda} \leq \tilde{x}$ such that

$$
T\left(\frac{1}{l A}, \tilde{x}\right)(t)=\tilde{x}(t), \quad T\left(\lambda, x_{\lambda}\right)(t)=x_{\lambda}(t), \quad t \in[0,1] .
$$

If $x_{\lambda}=\tilde{x}$, we have

$$
T\left(\lambda, x_{\lambda}\right)=x_{\lambda}=\tilde{x}=T\left(\frac{1}{l A}, \tilde{x}\right)=T\left(\frac{1}{l A}, x_{\lambda}\right) .
$$

This contradiction shows that $x_{\lambda}<\tilde{x}$. 
Define $\Omega_{1}=\{x \in E:-r<x(t)<\tilde{x}(t), t \in[0,1]\}$, where $r>0$ is the same as in the first part of Theorem 1. For any $x \in P \cap \partial \Omega_{1}$, we obtain $\|x\|=\|\tilde{x}\|$, and

$$
\|T(\lambda, x)\|<\frac{1}{l A} \int_{0}^{1} G(0, s) g(s) f(\tilde{x}(s)) d s=\tilde{x}(0)=\|\tilde{x}\| .
$$

Therefore,

$$
\|T(\lambda, x)\|<\|x\|, \quad x \in P \cap \partial \Omega_{1} .
$$

As in the proof in Theorem 1, there is $R>0$ large enough such that

$$
\|T(\lambda, x)\|>\|x\|, \quad x \in P \cap \partial \Omega_{2},
$$

where $\Omega_{2}=\{x \in E:\|x\|<R\}$. By compression expansion fixed point theorem, we see that $T$ has a fixed point $\bar{x}_{\lambda} \in P \cap\left(\Omega_{2} \backslash \bar{\Omega}_{1}\right)$. Since $x_{\lambda} \in \Omega_{1}, x_{\lambda} \neq \bar{x}_{\lambda}$, problem (1) has a second positive solution.

Theorem 3. Assume that $\left(H_{1}\right)$ and $\left(H_{2}\right)$ hold, and that $f(0)>0$. Then, for any $\lambda \in(0, \infty), B V P$ (1) admits a unique positive solution $\dot{x}_{\lambda}(t)$, and $\dot{x}_{\lambda}(t)$ satisfies:

(i) $\dot{x}_{\lambda}(t)$ is nondecreasing with respect to $\lambda$;

(ii) $\lim _{\lambda \rightarrow 0^{+}}\left\|\dot{x}_{\lambda}\right\|=0, \lim _{\lambda \rightarrow \infty}\left\|\dot{x}_{\lambda}\right\|=\infty$;

(iii) $\left\|\dot{x}_{\lambda}-\dot{x}_{\lambda_{0}}\right\| \rightarrow 0$ as $\lambda \rightarrow \lambda_{0}$.

Proof. Since $T$ is nondecreasing, for $u \in P$, we have

$$
T(\lambda, \kappa x)(t) \geq \phi(\kappa) \lambda \int_{0}^{1} G(t, s) g(s) f(x(s)) d s=\phi(\kappa) T(\lambda, x)(t), \quad t \in[0,1] .
$$

Denote

Define $\widehat{x}(t)=K_{\lambda}$, where $K_{\lambda}$ is given by Lemma 6, then $\widehat{x} \in P$ and $V K_{\lambda} \leq T(\lambda, \widehat{x})(t) \leq U_{x} K_{\lambda}$.

$$
\bar{V}=\sup \left\{\mu: \mu K_{\lambda} \leq T(\lambda, \widehat{x})(t)\right\}, \quad \bar{U}=\inf \left\{\mu: \mu K_{\lambda} \geq T(\lambda, \widehat{x})(t)\right\},
$$

then $\bar{V} \geq V$ and $\bar{U} \leq U_{x}$. Select $\widetilde{V}$ and $\widetilde{U}$ so that

$$
0<\widetilde{V}<\min \left\{1, F^{-1}(\bar{V})\right\}, \quad 0<\frac{1}{\widetilde{U}}<\min \left\{1, F^{-1}\left(\frac{1}{\bar{U}}\right)\right\} .
$$

We define

$$
\begin{array}{ll}
x_{1}(t)=\widetilde{V} K_{\lambda}, x_{k+1}(t)=T\left(\lambda, x_{k}\right)(t), \quad t \in[0,1], k=1,2, \cdots, \\
y_{1}(t)=\widetilde{U} K_{\lambda}, y_{k+1}(t)=T\left(\lambda, y_{k}\right)(t), \quad t \in[0,1], k=1,2, \cdots .
\end{array}
$$

Combining the properties of $T$ and (4), we get

$$
\widetilde{V} K_{\lambda}=x_{1}(t) \leq x_{2}(t) \leq \cdots \leq x_{k}(t) \leq \cdots \leq y_{k}(t) \leq \cdots \leq y_{2}(t) \leq y_{1}(t)=\widetilde{U} K_{\lambda} .
$$

Let $d=\frac{\widetilde{V}}{\widetilde{U}^{\prime}}$, obviously $0<d<1$. We claim that

$$
x_{k}(t) \geq \phi_{k-1}(d) y_{k}(t), \quad t \in[0,1], k=1,2, \cdots,
$$

where $\phi_{0}(d)=d, \phi_{k}(d)=\phi\left(\phi_{k-1}(d)\right), k=1,2, \cdots$. In fact, $x_{1}(t)=d y_{1}(t)=\phi_{0}(d) y_{1}(t), t \in[0,1]$. Suppose $x_{n}(t) \geq \phi_{n-1}(d) y_{n}(t)$ for $t \in[0,1]$, then

$$
x_{n+1}(t) \geq T\left(\lambda, \phi_{n-1}(d) y_{n}\right)(t) \geq \phi\left(\phi_{n-1}(d)\right) T\left(\lambda, y_{n}\right)(t)=\phi_{n}(d) y_{n+1}(t) .
$$


Hence, it follows by induction that (6) is true. According to (5) and (6), one has

$$
0 \leq x_{n+m}(t)-x_{n}(t) \leq y_{n}(t)-x_{n}(t) \leq\left(1-\phi_{n-1}(d)\right) y_{1}(t)=\left(1-\phi_{n-1}(d)\right) \widetilde{U} K_{\lambda},
$$

where $m \geq 0$ is an integer. Thus,

$$
\left\|x_{n+m}-x_{n}\right\| \leq\left\|y_{n}-x_{n}\right\| \leq\left(1-\phi_{n-1}(d)\right) \widetilde{U} K_{\lambda} .
$$

We claim that $\lim _{n \rightarrow \infty} \phi_{n}(d)=1$. From $\left(H_{2}\right)$ and $0<d<1$, we see that $\phi(d) \in(d, 1)$ and $d=\phi_{0}(d)<\phi_{1}(d)<\cdots<\phi_{n}(d)<\cdots<1$. Sequence $\left\{\phi_{n}(d)\right\}_{n=1}^{\infty}$ is increasing and bounded, there is $p \in[d, 1]$ such that $\lim _{n \rightarrow \infty} \phi_{n}(d)=p$. By the continuity of $\phi$ and $\phi_{n}(d)=\phi\left(\phi_{n-1}(d)\right)$, we conclude that $p=\phi(p)$, i.e., $F(p)=1$. It follows that $p=1$. Inequality (7) implies that there exists $\bar{x} \in P$ such that $\lim _{n \rightarrow \infty} x_{n}(t)=\lim _{n \rightarrow \infty} y_{n}(t)=\bar{x}(t)$ for $t \in[0,1]$. Clearly, $\bar{x}(t)$ is a positive solution of problem (1).

Suppose that $\bar{x}_{1}(t)$ and $\bar{x}_{2}(t)$ are positive solutions of problem (1), then $T\left(\lambda, \bar{x}_{1}\right)(t)=\bar{x}_{1}(t)$ and $T\left(\lambda, \bar{x}_{2}\right)(t)=\bar{x}_{2}(t), t \in[0,1]$. Define $\tilde{\delta}=\sup \left\{\delta: \bar{x}_{1}(t) \geq \delta \bar{x}_{2}(t)\right\}$, then $\bar{x}_{1}(t) \geq \tilde{\delta} \bar{x}_{2}(t)$. We claim that $\tilde{\delta} \geq 1$. Otherwise, $\tilde{\delta}<1$. Assumption $\left(H_{2}\right)$ implies that $f\left(\tilde{\delta} \bar{x}_{2}(t)\right)>\varphi(\tilde{\delta}) f\left(\bar{x}_{2}(t)\right), t \in[0,1]$. Since $f$ is nondecreasing,

$$
\bar{x}_{1}(t)=T\left(\lambda, \bar{x}_{1}\right)(t) \geq T\left(\lambda, \tilde{\delta} \bar{x}_{2}\right)(t)>\phi(\tilde{\delta}) T\left(\lambda, \bar{x}_{2}\right)(t)>\tilde{\delta} \bar{x}_{2}(t), \quad t \in[0,1],
$$

a contradiction. Then, $\bar{x}_{1}(t) \geq \bar{x}_{2}(t)$ for $t \in[0,1]$. Similarly, $\bar{x}_{2}(t) \geq \bar{x}_{1}(t)$. Therefore, $\bar{x}_{1}(t)=\bar{x}_{2}(t), t \in$ $[0,1]$. This proves the uniqueness result.

Next, we show that $(i)-($ iii $)$ hold. Let

$$
(H x)(t)=\int_{0}^{1} G(t, s) g(s) f(x(s)) d s, \quad t \in[0,1],
$$

then $T(\lambda, x)=\lambda H x$. Since $P^{o}=\{x \in P: x(t)>0, t \in[0,1]\}$ is nonempty, the operator $H: P^{o} \rightarrow P^{o}$ is increasing, and $H(\kappa x) \geq \phi(\kappa) H x$ for $0<\kappa<1$. Let $\omega=\frac{1}{\lambda}$. We now write $H x_{\omega}=\omega x_{\omega}$ instead of $\lambda H x_{\lambda}=x_{\lambda}$. Assume $0<\omega_{1}<\omega_{2}$, then $x_{\omega_{1}} \geq x_{\omega_{2}}$. Indeed, denote $\bar{\omega}=\sup \left\{t>0: x_{\omega_{1}} \geq t x_{\omega_{2}}\right\}$, then $\bar{\omega} \geq 1$. Otherwise $0<\bar{\omega}<1$. Direct computations yield $\omega_{1} x_{\omega_{1}}=H x_{\omega_{1}} \geq H\left(\bar{\omega} x_{\omega_{2}}\right) \geq \phi(\bar{\omega}) H x_{\omega_{2}}=$ $\phi(\bar{\omega}) \omega_{2} x_{\omega_{2}}$, then $x_{\omega_{1}} \geq \frac{\omega_{2}}{\omega_{1}} \phi(\bar{\omega}) x_{\omega_{2}}>\bar{\omega} x_{\omega_{2}}$. This is a contradiction to the definition of $\bar{\omega}$. Thus, $\bar{\omega} \geq 1, x_{\omega_{1}} \geq x_{\omega_{2}}$, and further

$$
x_{\omega_{1}}=\frac{1}{\omega_{1}} H x_{\omega_{1}} \geq \frac{1}{\omega_{1}} H x_{\omega_{2}}=\frac{\omega_{2}}{\omega_{1}} x_{\omega_{2}} \gg x_{\omega_{2}}, \quad 0<\omega_{1}<\omega_{2} .
$$

Then, $x_{\omega}(t)$ is strong decreasing in $\omega$, that is, $x_{\lambda}(t)$ is strong increasing in $\lambda$. Let $\omega_{2}=\omega$ and fix $\omega_{1}$ in (8), for $\omega>\omega_{1}$, we have $x_{\omega_{1}} \geq \frac{\omega}{\omega_{1}} x_{\omega}$, and

$$
\left\|x_{\omega}\right\| \leq \frac{N \omega_{1}}{\omega}\left\|x_{\omega_{1}}\right\|
$$

where $N>0$ is a normal constant of cone $P$. Because $\omega=\frac{1}{\lambda}$, then $\lim _{\lambda \rightarrow 0^{+}}\left\|x_{\lambda}\right\|=0$. Let $\omega_{1}=\omega$ and fix $\omega_{2}$ in (8), we obtain $\lim _{\lambda \rightarrow+\infty}\left\|x_{\lambda}\right\|=+\infty$.

Finally, for given $\omega_{0}$, by $(8)$, we have

$$
x_{\omega} \ll x_{\omega_{0}}, \quad \omega>\omega_{0} .
$$

Let $t_{\omega}=\sup \left\{t>0: x_{\omega} \geq t x_{\omega_{0}}, \omega>\omega_{0}\right\}$, then $0<t_{\omega}<1$ and $x_{\omega} \geq t_{\omega} x_{\omega_{0}}$. Direct computations yield $\omega x_{\omega}=H x_{\omega} \geq H\left(t_{\omega} x_{\omega_{0}}\right) \geq \phi\left(t_{\omega}\right) H x_{\omega_{0}}=\phi\left(t_{\omega}\right) \omega_{0} x_{\omega_{0}}$. By the definition of $t_{\omega}$, we have $\frac{\omega_{0}}{\omega} \phi\left(t_{\omega}\right) \leq t_{\omega}$, and 


$$
t_{\omega} \geq F^{-1}\left(\frac{\omega_{0}}{\omega}\right), \quad \forall \omega>\omega_{0} .
$$

Combining (9) with (10), one has that

$$
\left\|x_{\omega_{0}}-x_{\omega}\right\| \leq N\left[1-F^{-1}\left(\frac{\omega_{0}}{\omega}\right)\right]\left\|x_{\omega_{0}}\right\| \rightarrow 0, \quad \omega \rightarrow \omega_{0}+0 .
$$

Similarly, $\left\|x_{\omega_{0}}-x_{\omega}\right\| \rightarrow 0, \omega \rightarrow \omega_{0}-0$. Hence, $\left\|x_{\omega_{0}}-x_{\omega}\right\| \rightarrow 0$ as $\omega \rightarrow \omega_{0}$.

Author Contributions: Both authors have contributed equally to this paper. Writing-original draft, X.H. and L.Z.; Writing-review and editing, X.H. and L.Z.

Funding: Supported financially by the National Natural Science Foundation of China $(11501318,11871302)$, the China Postdoctoral Science Foundation (2017M612230), the Natural Science Foundation of Shandong Province of China (ZR2017MA036) and the International Cooperation Program of Key Professors by Qufu Normal University.

Conflicts of Interest: The authors declare no conflict of interest.

\section{References}

1. Infante, G.; Webb, J.R.L. Loss of positivity in a nonlinear scalar heat equation. NoDEA Nonlinear Differ. Equ. Appl. 2006, 13, 249-261. [CrossRef]

2. Webb J.R.L. Multiple positive solutions of some nonlinear heat flow problems. Discret. Contin. Dyn. Syst. 2005, 2005, 895-903.

3. Guidotti, P.; Merino, S. Gradual loss of positivity and hidden invariant cones in a scalar heat equation. Differ. Integral Equ. 2000, 13, 1551-1568.

4. Webb, J.R.L. Existence of positive solutions for a thermostat model. Nonlinear Anal. Real World Appl. 2012, 13, 923-938. [CrossRef]

5. Webb, J.R.L. Remarks on a non-local boundary value problem. Nonlinear Anal. 2010, 72, 1075-1077. [CrossRef]

6. Infante, G. Positive solutions of nonlocal boundary value problems with singularities. Discret. Contin. Dyn. Syst. 2009, 2009, 377-384.

7. Infante, G.; Webb J.R.L. Nonlinear non-local boundary-value problems and perturbed Hammerstein integral equations. Proc. Edinb. Math. Soc. 2006, 49, 637-656. [CrossRef]

8. Palamides, P.K.; Infante, G.; Pietramala, P. Nontrivial solutions of a nonlinear heat flow problem via Sperner's lemma. Appl. Math. Lett. 2009, 22, 1444-1450. [CrossRef]

9. Infante, G.; Pietramala, P.; Tenuta, M. Existence and localization of positive solutions for a nonlocal BVP arising in chemical reactor theory. Commun. Nonlinear Sci. Numer. Simul. 2014, 19, 2245-2251. [CrossRef]

10. Cianciaruso, F.; Infante, G.; Pietramala, P. Solutions of perturbed Hammerstein integral equations with applications. Nonlinear Anal. Real World Appl. 2017, 33, 317-347. [CrossRef]

11. Calamai, A.; Infante, G. Nontrivial solutions of boundary value problems for second-order functional differential equations. Ann. Mat. Pura Appl. 2016, 195, 741-756. [CrossRef]

12. Infante, G.; Pietramala, P.; Tojo, F.A.F. Non-trivial solutions of local and non-local Neumann boundary-value problems. Proc. R. Soc. Edinb. Sect. A 2016, 146, 337-369. [CrossRef]

13. Cabada, A.; Infante, G.; Tojo, F.A.F. Nonzero solutions of perturbed Hammerstein integral equations with deviated arguments and applications. Topol. Methods Nonlinear Anal. 2016, 47, 265-287. [CrossRef]

14. Li, Z. Existence of positive solutions of superlinear second-order Neumann boundary value problem. Nonlinear Anal. 2010, 72, 3216-3221. [CrossRef]

15. Ma, R.; An, Y. Global structure of positive solutions for superlinear second order $m$-point boundary value problems. Topol. Methods Nonlinear Anal. 2009, 34, 279-290. [CrossRef]

16. Cahlon, B.; Schmidt, D.; Shillor, M.; Zou, X. Analysis of thermostat models. Eur. J. Appl. Math. 1997, 8, 437-455. [CrossRef]

17. Ji, D.; Bai, Z.; Ge, W. The existence of countably many positive solutions for singular multipoint boundary value problems. Nonlinear Anal. 2010, 72, 955-964. [CrossRef]

18. Nieto, J.J.; Pimentel, J. Positive solutions of a fractional thermostat model. Bound. Value Probl. 2013, 2013, 5. [CrossRef] 
19. Cabada, A.; Infante, G. Positive solutions of a nonlocal Caputo fractional BVP. Dyn. Syst. Appl. 2014, 23, 715-722.

20. Shen, C.; Zhou, H.; Yang, L. Existence and nonexistence of positive solutions of a fractional thermostat model with a parameter. Math. Methods Appl. Sci. 2016, 39, 4504-4511. [CrossRef]

21. Senapati, T.; Dey, L.K. Relation-theoretic metrical fixed-point results via $w$-distance with applications. J. Fixed Point Theory Appl. 2017, 19, 2945-2961. [CrossRef]

22. Cabrera, I.J.; Rocha, J.; Sadarangani, K.B. Lyapunov type inequalities for a fractional thermostat model. Rev. Real Acad. Cienc. Exactas Fis. Nat. Ser. A Math. 2018, 112, 17-24. [CrossRef]

23. Hao, X.; Sun, H.; Liu L. Existence results for fractional integral boundary value problem involving fractional derivatives on an infinite interval. Math. Methods Appl. Sci. 2018, 41, 6984-6996. [CrossRef]

24. Hao, X. Positive solution for singular fractional differential equations involving derivatives. Adv. Differ. Equ. 2016, 2016, 139. [CrossRef]

25. Graef, J.R.; Kong, L.; Wang, H. Existence, multiplicity, and dependence on a parameter for a periodic boundary value problem. J. Differ. Equ. 2008, 245, 1185-1197. [CrossRef]

26. Hao, X.; Wang, H. Positive solutions of semipositone singular fractional differential systems with a parameter and integral boundary conditions. Open Math. 2018, 16, 581-596. [CrossRef]

27. Novozhenova, O.G. Life and science of Alexey N. Gerasimov. On the linear operators, elastic viscosity, elevterous and fractional derivatives. arXiv 2018, arXiv:1808.04397.

28. Samko, S.G.; Kilbas, A.A.; Marichev, O.I. Fractional Integrals and Derivatives: Theory and Applications; Gordon and Breach: New York, NY, USA, 1993.

29. Kilbas, A.A.; Srivastava, H.M.; Trujillo, J.J. Theory and Applications of Fractional Differential Equations; Elsevier: Amsterdam, The Netherlands, 2006.

30. Kiryakova, V. Generalized Fractional Calculus and Applications; Longman: Harlow, UK, 1994.

31. Podlubny, I. Fractional Differential Equations; Academic Press: New York, NY, USA, 1999.

32. Guo, D.; Lakshmikantham, V. Nonlinear Problems in Abstract Cones; Academic Press: Boston, MA, USA, 1988.

(C) 2019 by the authors. Licensee MDPI, Basel, Switzerland. This article is an open access article distributed under the terms and conditions of the Creative Commons Attribution (CC BY) license (http://creativecommons.org/licenses/by/4.0/). 